\title{
Lesiones nodulares en un neonato
}

\author{
Nodular lesions in a newborn
}

\author{
Aniza Giacamana, Marco Antonio Martínez Ortega ${ }^{\mathrm{b}}$, Julián Boix Vilanova ${ }^{\mathrm{a}}$, \\ Carlos Saus Sarrias ${ }^{b}$, Teresa Carrión Mera ${ }^{c}$, Ana Martín-Santiago ${ }^{a}$
}

\author{
aDepartamento de Dermatología, Hospital Universitari Son Espases, Palma de Mallorca, España

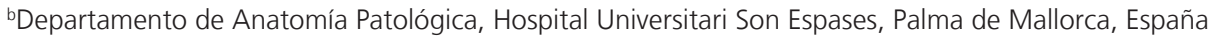 \\ ‘Departamento de Pediatría, Hospital Universitari Son Espases, Palma de Mallorca, España
}

Recibido: 24 de septiembre de 2018; Aprobado: 24 de diciembre de 2018

Palabras clave: Necrosis grasa; recién nacido; paniculitis; nódulos; hipercalcemia.

Key words: Fat necrosis; newborn; panniculitis, nodules; hypercalcemia.

\section{Caso clínico}

La necrosis grasa subcutánea del recién nacido es una entidad poco frecuente de curso benigno y autolimitado, producida por una paniculitis del tejido celular subcutáneo. Se observa en pacientes nacidos a término o postérmino, que en general han estado sometidos a factores estresantes durante el periodo perinatal. Es importante vigilar los niveles de calcio ya que se puede asociar a hipercalcemia y provocar daño renal si no se realiza un tratamiento oportuno.

Presentamos el caso clínico de un neonato de 20 días de vida, remitido a nuestro centro para la valoración de lesiones nodulares de 10 días de evolución, con el diagnóstico de abscesos que persistían tras completar tratamiento antibiótico intravenoso. Producto de una gestación controlada. La madre sin antecedentes de consumo de drogas, presentó preeclamsia durante la segunda mitad del embarazo por lo que el parto fue inducido a las 39 semanas, requiriendo el uso de fórceps por distocia de hombros. El peso al nacer fue 4.275 gr, con un Apgar de 4-7, precisando reanimación con mascarilla. A la exploración física era un paciente fototipo VI, en el que destacaban nódulos de color violáceo-marrón indurados, dolorosos a la palpación y no adheridos a planos profundos de entre 2 y $3.5 \mathrm{~cm}$ de diámetro, localizados en la región occipital, submaxilar (figura 1), cervical izquierda, y uno de morfología lineal en la región frontal derecha (figura 2). Las lesiones no presentaban frémito, pulso, ni aumento de la temperatura local. Su estado general era bueno y no se observaron alteraciones en los reactantes de fase aguda ni en las pruebas de coagulación.

Con la sospecha diagnóstica de adenopatías, metástasis, sarcomas, hemangiomas, angioma en penacho y hemangioendotelioma Kaposiforme, se realizó una biopsia de piel. En el estudio histológico se observó una paniculitis lobulillar (figura 3a), con espículas de disposición radial en el interior de los adipocitos, y un denso infiltrado linfohistiocitario, con células gigantes multinucleadas y eosinófilos (figura 3b), por lo que se diagnosticó una necrosis grasa subcutánea del recién nacido. 


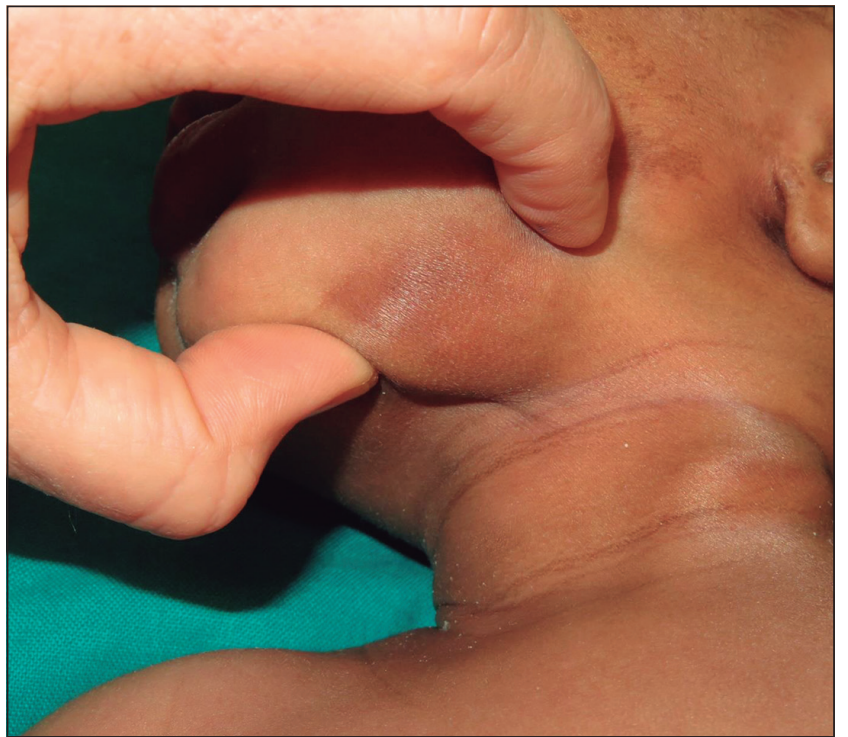

Figura 1. Nódulo indurado submandibular.

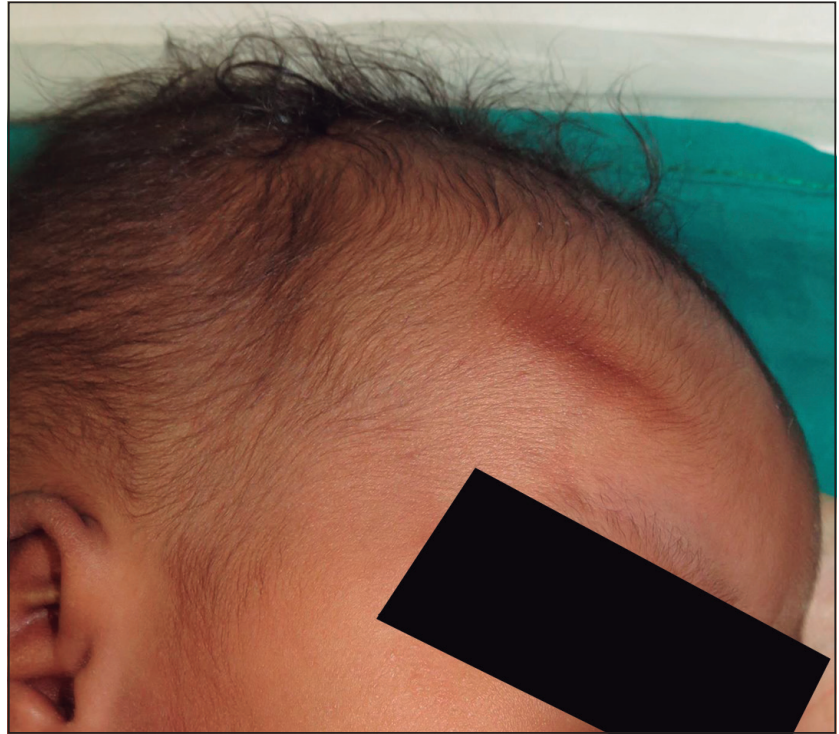

Figura 2. Placa de morfología lineal en región frontal.

Figura 3. (a) Paniculitis Iobulillar. (b) Espículas de disposición radial dentro de los adipocitos.
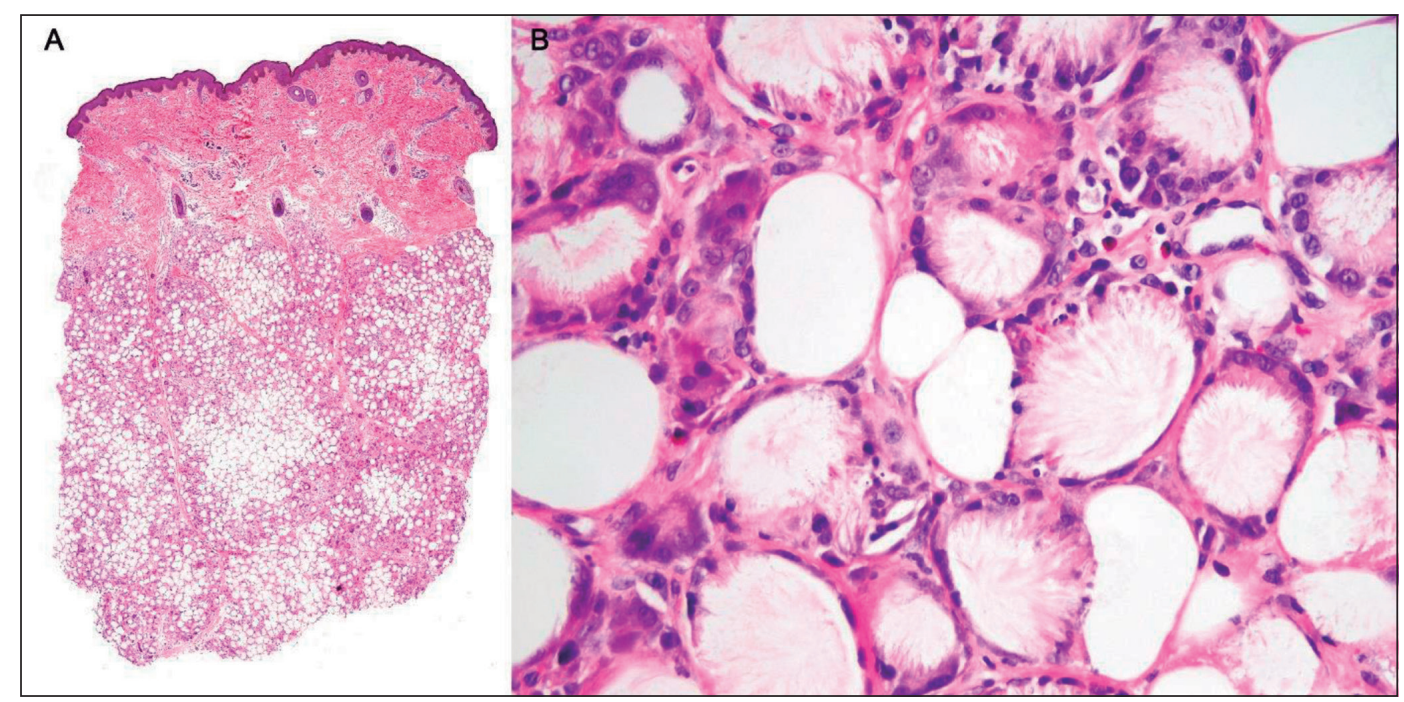

\section{Comentario}

La necrosis grasa subcutánea del recién nacido (NGSRN) es una paniculitis poco frecuente que se manifiesta durante las primeras semanas de vida en recién nacidos a término o post-término. Clínicamente se caracteriza por la aparición de placas o nódulos únicos o múltiples de color eritematoso o violáceo, de bordes bien definidos, indurados al tacto y no adheridos a planos profundos, con o sin aumento de la temperatura local, y que se localizan más frecuentemente en mejillas, región dorsal, brazos, nalgas y muslos ${ }^{1-4}$.

La etiopatogenia de esta entidad es desconocida, aunque parece estar en relación con la composición de la grasa subcutánea de los neonatos, que presenta una concentración relativamente alta de ácidos grasos saturados, ricos en aminoácidos palmítico y esteárico. Estos ácidos grasos, tienen un punto de fusión más alto $\left(64^{\circ} \mathrm{C}\right)$, lo que los hace más propensos a precipitar a bajas temperaturas, provocando la necrosis de los adipocitos. Otros mecanismos propuestos incluyen una inmadurez de los sistemas enzimáticos involucrados en el metabolismo de los ácidos grasos e hipoxia de la grasa subcutánea ${ }^{1,2}$.

Factores predisponentes son diabetes mellitus materna o diabetes gestacional, preeclampsia, tabaquismo, uso de cocaína o bloqueadores de los canales de calcio durante el embarazo, así como la hipotermia y cirugía cardiaca del neonato, infecciones y traumas cutáneos ${ }^{2,4}$.

El diagnóstico de la NGSRN se confirma mediante la 
biopsia de piel, donde se observan áreas de paniculitis lobulillar, con cristales birrefringentes de disposición radial desde el centro hacia la periferia de los adipocitos. El infiltrado inflamatorio puede estar compuesto por linfocitos, histiocitos, células gigantes multinucleadas y eosinófilos ${ }^{1,2}$.

El diagnóstico diferencial se plantea con el esclerema neonatorum que suele ocurrir en recién nacidos a pretérmino y gravemente enfermos. En los que se observa un empastamiento cutáneo de color amarillento, rápidamente progresivo y con una elevada tasa de mortalidad. ${ }^{3}$ La NGSRN también se confunde con celulitis, adenopatías, eritema nodoso, histiocitosis, tumores benignos como el hemangioma infantil y tumores malignos como el sarcoma, neuroblastoma o leucemias.

Dentro de las complicaciones se incluyen hipercalcemia, dislipemia, insuficiencia renal, atrofia subcutánea y en casos aislados calcificaciones metastásicas en riñones, piel, miocardio, hígado, vena cava y mucosa gástrica ${ }^{2,4}$. En un estudio se observó que un 63\% de pacientes con NGSRN desarrollaron hipercalcemia, en la mayoría de los casos leve. ${ }^{4} \mathrm{El}$ manejo de la hipercalcemia incluye restricciones dietéticas, hiperhidratación, diuréticos calciuricos, corticoides y en casos más resistentes el uso de bifosfonatos ${ }^{6}$.

El pronóstico de la NGSRN es bueno y en general ocurre una regresión completa de las lesiones en semanas o meses, aunque se ha descrito el tratramiento quirúrgico en lesiones persistentes y de gran tamaño ${ }^{7}$. Es importante el seguimiento clínico y de la hipercalcemia durante entre 2 y 6 meses tras el inicio de las lesiones cutáneas, aunque la evidencia publicada hasta el momento se basa solamente en casos clínicos, revisiones sistemáticas de la literatura y en la opinión de expertos ${ }^{2,3,8}$.

Nuestro paciente presentó una hipercalcemia leve, que se resolvió a los pocos días de iniciar una dieta con bajos aportes de calcio y vitamina D. Las cifras de calcio se han mantenido dentro de los límites de la normalidad, y los nó- dulos se han resuelto por completo tras 2 meses sin requerir tratamiento, por lo que actualmente sigue una dieta normal.

\section{Conclusión}

Presentamos el caso de un neonato con antecedente de preeclampsia materna y parto instrumentado con fórceps, que desarrolló lesiones nodulares de predominio en la región craneocervical, en donde el diagnóstico diferencial fue amplio y en el que el estudio histológico confirmó el diagnóstico de NGSRN, lo que permitió realizar un adecuado seguimiento y tratamiento de la hipercalcemia asociada.

\section{Responsabilidades Éticas}

Protección de personas y animales: Los autores declaran que los procedimientos seguidos se conformaron a las normas éticas del comité de experimentación humana responsable y de acuerdo con la Asociación Médica Mundial y la Declaración de Helsinki.

Confidencialidad de los datos: Los autores declaran que han seguido los protocolos de su centro de trabajo sobre la publicación de datos de pacientes.

Derecho a la privacidad y consentimiento informado: Los autores han obtenido el consentimiento informado de los pacientes y/o sujetos referidos en el artículo. Este documento obra en poder del autor de correspondencia.

\section{Conflicto de intereses}

Los autores declaran no tener conflicto de intereses.

\section{Referencias}

1. Torrelo A, Hernández A. Panniculitis in Children. Dermatologic Clinics. 2008;26:491-500.

2. Mahé E, Girszyn N, Hadj-Rabia S, Bodemer C, Hamel-Teillac D, De Prost Y. Subcutaneous fat necrosis of the newborn: a systematic evaluation of risk factors, clinical manifestations, complications and outcome of 16 children. Br J Dermatol [Internet]. 2007;156:709-15.

3. Cameselle D, Islas D, Montenegro T, Afonso JL, Hernández B. Nódulos subcutáneos en un recién nacido. Actas Dermosifiliogr. 2006;97:218-20.

4. Del Pozzo-Magaña BR, Ho N. Subcutaneous Fat Necrosis of the Newborn: A 20-Year Retrospective Study. Pediatr Dermatol. 2016;33:e353-5.

5. Lara LG, Villa AV, Rivas MMO, Capella MS, Prada F, Enseñat MAG. Subcutaneous Fat Necrosis of the Newborn: Report of Five Cases. Pediatr Neonatol [Internet]. 2014; Available from: http://www.sciencedirect.com/science/ article/pii/S1875957214001685

6. Samedi VM, Yusuf K, Yee W, Obaid H, Al Awad EH. Neonatal hypercalcemia secondary to subcutaneous fat necrosis successfully treated with pamidronate: A case series and literature review. AJP Rep. 2014;4:e93-6.

7. Beuzeboc Gérard M, Aillet S, Bertheuil N, Delliere V, Thienot S, Watier E. Surgical management of subcutaneous fat necrosis of the newborn required due to a lack of improvement: A very rare case. $\mathrm{Br} \mathrm{J}$ Dermatol. 2014;171:183-5.

8. Stefanko NS, Drolet BA. Subcutaneous fat necrosis of the newborn and associated hypercalcemia: A systematic review of the literature. Pediatr Dermatol. 2018;00:1-7. 